\title{
A Two Hop Relay Receiving Method using LTE Transmission Technology
}

\author{
Ziqiang Chen, Yunxiao Zu, Weihai Li, Bin Hou \\ School of Electronic Engineering Beijing University of Posts and Telecommunications, Beijing 100876, China \\ \{zq.chenmail\& houbin23\}@gmail.com, \{zuyx\& liweihai\}@bupt.edu.cn
}

\begin{abstract}
Aiming at the two hop relay’s MIMO(Multiple Input Multiple Output) transmission, this paper studied the two hops relay MIMO receiving model which is applicable to these situations: different data stream on the different antenna in the transmitter, different noise power in the transmit channel and SSD(Soft Sphere Decode) in the receiver; And developed a receiving method of the relay link signal and the direct link signal in this model, which is for the purpose of maximum SNR(Signal to Noise Ratio). The BER curves of relay user under different receiving schemes are obtained through simulation. The conclusions are that the developed receiving method is superior to the selective diversity combination algorithm, and it has a considerable robustness to combine signals from different noise power channel.
\end{abstract}

Index Terms - Receiving Method, Two Hop Relay, LTE (Long Term Evolution)

\section{Introduction}

With the completion of LTE protocol, 3GPP (The $3^{\text {rd }}$ Generation Partnership Project) entered a new stage of LTE-A (LTE Advanced) for further communication performance. Evolving from LTE, LTE-A extended the MIMO technique, introduced relay transmission and other new technologies [1]. Relay technology can effectively improve remote users' communication, and the details are still under discussion [2]. One of the hot fields of relay technology is two hop relay based on time division multiplexing. As to relay user supported by two hop LTE relay, there are two downlink signals when receiving, namely, direct link signal and access link signal. Thus utilizing both signals to combine them to receive information is helpful for relay users.

The main methods to receive signals by combing them are selective combination, equal gain combination and MRC(Maximum Ratio Combination) [3]. MRC can achieve the Maximization of signal to noise ratio, help to demodulating signals, this method is proposed in [4].

Recent studies of relay MIMO transmission have these characteristics: each antenna of MIMO transmitter has same transmission information, the different transmit link has same power of interference noise and the demodulation of relay MIMO signal is mostly hard demodulation [1][2][5]. But these situations are not practical when relay transmit using LTE technology as LTE MIMO tech supports different antenna transmitting different signal, receiver demodulation using SSD (Soft Sphere Demodulation) [6] and direct link and access link may have different noise power. Thus this paper is dealing with the above two hop LTE relay MIMO transmission receiving situation.

\section{Introduction Signal Model and Receiving Method}

A. Signal Model

The two hop LTE relay wireless transmission network topology is show below in Fig.1.

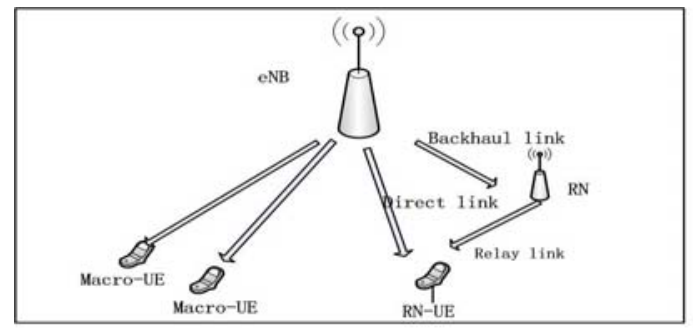

Fig. 1 The studied wireless relay transmission network topology

In Fig.1, eNB represents the evolved Node Base, RN means Relay Node, Macro-UE means the Macro User Equipment, RN-UE means Relay user. In this scenario, we suppose backhaul link channel situation is good enough to ensure the $100 \%$ right reception of RN [7]. RN-UE transmission signals on direct link and relay link have the same coding, modulation, MIMO and resource assignment schemes, with means the signals part of the same RN-UE are identical [8].

As we concentrating on relay user's receiving method, the signal model on RN-UE's receiving side is depict in Fig.2.

As mentioned before, the signal of one relay user on access link and direct link are same, the signals in Fig.2 of direct link transmitter and access link on different signal are both depicted by the vector $S_{1}, S_{2}, \ldots, S_{T}$, . The signal dimensions on each antenna are equally $1 \times L$ ( $\mathrm{L}$ is the signal length). The signal relay user receiving contains access link

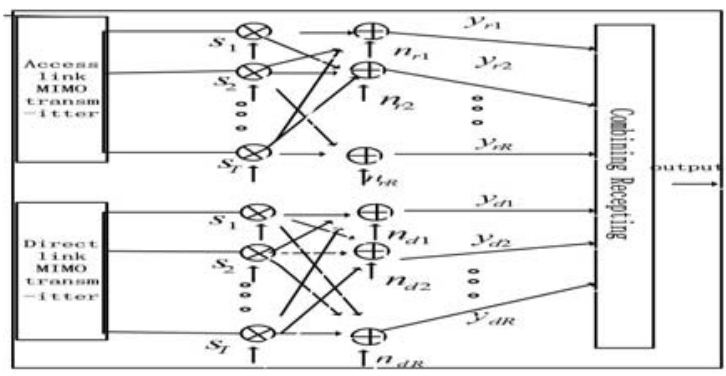

Fig. 2 Signal processing model on RN-UE’s receiving side. 
$Y_{d}$ (dimension of $R \times L$ )signal and direct link signal $Y_{r}$ (dimension of $R \times L$ ). $T$ and $R$ represent transmit and receive antenna port amount respectively. So $Y_{d}$ and $Y_{r}$ both consist of $R$ branches of sub-signal, seen in (1).

$$
\begin{gathered}
Y_{r}=H S+N_{r}=\left[\begin{array}{llll}
y_{r 1} & y_{r 2} & \cdots & y_{r R}
\end{array}\right]^{\mathrm{T}}, Y_{d}=G S+N_{d}=\left[\begin{array}{lll}
y_{d 1} & y_{d 2} \cdots y_{d R}
\end{array}\right]^{\mathrm{T}} \\
S=\left[s_{1}, s_{2}, \cdots, s_{T}\right]^{T}
\end{gathered}
$$

$H$ and $G$ are access link channel response matrix and direct link channel response respectively.

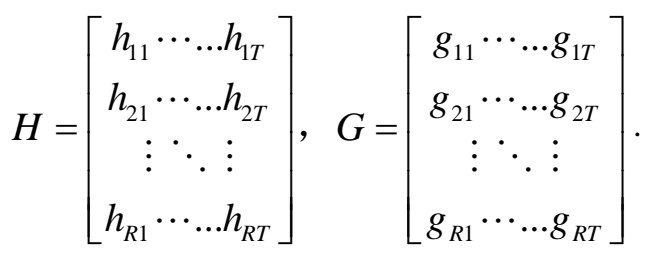

$\boldsymbol{N}_{r}$ and $\boldsymbol{N}_{d}$ are access link noise and direct link noise, they are both white Gauss noise and can be both express as matrix of dimension $R \times L$ :

$$
N_{r}=\left[n_{r 1}, n_{r 2}, \ldots n_{r R}\right]^{\mathrm{T}}, N_{d}=\left[n_{d 1}, n_{d 2}, \ldots n_{d R}\right]^{\mathrm{T}} .
$$

In (4), $\boldsymbol{n}_{r 1}, \boldsymbol{n}_{r 2}, \ldots \boldsymbol{n}_{r R}, \boldsymbol{n}_{d 1}, \boldsymbol{n}_{d 2}, \ldots \boldsymbol{n}_{d R}$ are sub branch noise of dimension $1 \times L$, they may have different power.

\section{B. Receiving Method}

As the sub branch signal on each antenna may be different, it need to applicable for SSD, in order to maximize SNR, we realize combining reception of direct link signal and access link signal based on each antenna port. The receiving method contains two parts, constructing the signal model based on each antenna port and accomplishing MRC combining reception port by port.

a. Constructing the signal model based on each antenna As the sub branch signal on each antenna may be different, but the sub branch signal $s_{i}(i=1, \ldots, R)$ on direct link and access link of same receiving antenna port number $i$ are same. We will combine the same antenna port signal on access and direct link (shown in Fig.2). Thus we constructing new signal model base on each receiving antenna port $i$. For receiving antenna port 1 , the model is below in (5) and (6):

$$
Y_{1}=\left[\begin{array}{ll}
y_{d 1} & y_{r 1}
\end{array}\right]^{\mathrm{T}}=\left[\begin{array}{c}
\sum_{T} h_{1 i} s_{i} \\
\sum_{T}^{T} g_{1 i} s_{i}
\end{array}\right]+\left[\begin{array}{c}
n_{d 1} \\
n_{r 1}
\end{array}\right]=\left[\begin{array}{c}
H_{1} \\
G_{1}
\end{array}\right] \cdot S+\left[\begin{array}{c}
n_{d 1} \\
n_{r 1}
\end{array}\right]=K_{1} S+N_{1} \text { (5) }
$$

$\boldsymbol{Y}_{1}$ means the receiving signal according to port 1 consisted of access and direct link signal. $\boldsymbol{K}_{1}$ is equivalent channel response( seen in (6)), and $\boldsymbol{N}_{1}=\left[\begin{array}{ll}\boldsymbol{n}_{d 1} & \boldsymbol{n}_{r 1}\end{array}\right]^{\mathrm{T}}$ is equivalent noise matrix. $\boldsymbol{n}_{d 1}, \boldsymbol{n}_{r 1}$ may has different power.

$$
K_{1}=\left[\begin{array}{l}
H_{1} \\
G_{1}
\end{array}\right]=\left[\begin{array}{llll}
h_{11} & h_{12} & \ldots & h_{1 T} \\
g_{11} & g_{12} & \ldots & g_{1 T}
\end{array}\right]
$$

b. MRC receiving according to each antenna port

Still take port 1 for example to depict the MRC receiving according to each antenna port. The receiving process can be express as (7). $\boldsymbol{R}_{1}$ is the combined signal and $\boldsymbol{w}_{1}$ is the combining vector.

$$
R_{1}=w_{1} Y_{1} .
$$

As $\boldsymbol{n}_{d 1}, \boldsymbol{n}_{r 1}$ may has different power, in order to accomplish MRC receiving [4], a efficient way is to transform the access link and direct link noise in to same power. So we first accomplish noise power transform and then MRC combination.

(1) Noise power transform

By $\boldsymbol{N}_{1}=\left[\begin{array}{ll}\boldsymbol{n}_{d 1} & \boldsymbol{n}_{r 1}\end{array}\right]^{\mathrm{T}}$, suppose the power of branch $\boldsymbol{n}_{d 1}, \boldsymbol{n}_{r 1}$ are depict by $a_{1}$ and $a_{2}$, we construct a transform matrix $\boldsymbol{T}$ :

$$
\boldsymbol{T}=\left[\begin{array}{cc}
\sqrt{a_{1}} & 0 \\
0 & \sqrt{a_{2}}
\end{array}\right] .
$$

According to our assumption, there must exists a noise matrix $\boldsymbol{N}_{t 1}$ whose branch noise power are same and satisfies:

$$
\boldsymbol{N}_{1}=\boldsymbol{T} \boldsymbol{N}_{t 1} \text {. }
$$

And under this situation, (5) and (7) turn to (10) and (11).

$$
\begin{gathered}
\boldsymbol{Y}_{1}=\boldsymbol{K}_{1} \boldsymbol{S}+\boldsymbol{T} \boldsymbol{N}_{t 1} . \\
\boldsymbol{R}_{1}=\boldsymbol{w}_{1}\left(\boldsymbol{K}_{1} \boldsymbol{S}+\boldsymbol{T} \boldsymbol{N}_{t 1}\right)=\boldsymbol{w}_{1} \boldsymbol{T}\left(\boldsymbol{T}^{-1} \boldsymbol{K}_{1} \boldsymbol{S}+\boldsymbol{T}^{-1} \boldsymbol{N}_{1}\right) . \\
=\boldsymbol{w}_{t 1}\left(\boldsymbol{K}_{t 1} \boldsymbol{S}+\boldsymbol{N}_{t 1}\right)=\boldsymbol{w}_{t 1} \boldsymbol{Y}_{t 1}
\end{gathered}
$$

And $\quad \boldsymbol{Y}_{t 1}=\boldsymbol{K}_{t 1} \boldsymbol{S}+\boldsymbol{N}, \boldsymbol{K}_{t 1}=\boldsymbol{T}^{-1} \boldsymbol{K}_{1}, \boldsymbol{w}_{t 1}=\boldsymbol{w}_{1} \boldsymbol{T}$.

(2) Same branch noise power MRC processing

If deal the branch sub signal with same noise power MRC in [4]. The processed signal (or combined signal) has the maximum signal to noise ratio property. Shown in (13).

$$
\boldsymbol{R}_{1}=\boldsymbol{w}_{t 1} \boldsymbol{Y}_{t 1}=\boldsymbol{w}_{t 1}\left(\boldsymbol{K}_{t 1} \boldsymbol{S}+\boldsymbol{N}_{t 1}\right) .
$$

The average power of useful signal in $\boldsymbol{R}_{1}$ can be calculated in (14).

$$
P_{\text {Aver }}=E\left[\left\|\boldsymbol{w}_{t 1} \boldsymbol{K}_{t 1} \boldsymbol{S}\right\|\right]=E\left[\boldsymbol{S}^{\mathrm{H}}\left(\boldsymbol{K}_{t 1}{ }^{\mathrm{H}} \boldsymbol{K}_{t 1} \boldsymbol{v}_{1}\right)\left(v_{1}^{\mathrm{H}} \boldsymbol{K}_{t 1}^{\mathrm{H}} \boldsymbol{K}_{t 1}\right) \boldsymbol{S}\right] .
$$

In (14), $\boldsymbol{v}_{1}$ is the vector satisfying $\boldsymbol{w}_{\mathrm{t} 1}=\left(\boldsymbol{K}_{\mathrm{t} 1} \boldsymbol{v}_{1}\right)^{\mathrm{H}}$. According to [11], when $\boldsymbol{v}_{1}=\left[v_{11}, v_{12} \ldots v_{1 T}\right]^{\mathrm{T}}$ is the 
normalized eigenvector of $\boldsymbol{K}_{t 1}{ }^{\mathrm{H}} \boldsymbol{K}_{t 1}$ at same time, (14) can be step into (15).

$$
P_{\text {Aver }}=E\left[\boldsymbol{S}^{\mathrm{H}} \lambda_{1} \boldsymbol{v}_{1} \boldsymbol{v}_{1}{ }^{\mathrm{H}} \lambda_{1} \boldsymbol{S}\right]=\lambda_{1}{ }^{2} E\left[\left\|\boldsymbol{S}^{\mathrm{H}} \boldsymbol{v}_{1}\right\|^{2}\right] .
$$

As in the paper, $\boldsymbol{S}$ is suppose to be QAM signal modulated from individual 0,1 bit sequence, elements (value) of $\boldsymbol{S}$ are independent, having mean value of 0 and their average power are equal(determined by QAM modulation scheme). Suppose the average power of the elements is $E_{s}$, then $P_{\text {Aver }}$ is (16).

$$
P_{\text {Aver }}=\lambda_{1}^{2} E\left[\left\|\boldsymbol{S}^{\mathrm{H}} \boldsymbol{v}_{1}\right\|^{2}\right]=\lambda_{1}^{2}\left(\sum_{i=1}^{T}\left\|v_{1 i}\right\|^{2}\right) E\left[\left\|\boldsymbol{s}_{i}\right\|^{2}\right]=\lambda_{1}^{2} E_{s} .
$$

Similarly, the average power $P_{N}$ of noise in $\boldsymbol{R}_{1}$ can be express by (17).

$$
P_{N}=E\left[\left\|\boldsymbol{w}_{t 1} \boldsymbol{N}_{t 1}\right\|\right]=\lambda_{1} E\left[\left\|\boldsymbol{N}_{t 1}{ }^{\mathrm{H}} \boldsymbol{v}_{1}\right\|^{2}\right]=\lambda_{1} \sigma^{2} .
$$

$\sigma^{2}$ is the average power of normalized noise $N_{t 1}$ (here satisfying $\sigma^{2}=1$ ). After combination process in (13), the maximum signal to noise ratio of $\boldsymbol{R}_{1}$ can be written in (18).

$$
S N R=\frac{P_{\text {Aver }}}{P_{N}}=\frac{\lambda_{1}^{2} E_{s}}{\lambda_{1} \sigma^{2}}=\lambda_{1} \cdot \frac{E_{s}}{\sigma^{2}} \Rightarrow S N R_{\max }=\lambda_{1 \max } \cdot \frac{E_{s}}{\sigma^{2}} .
$$

As the average signal power $P_{\text {Aver }}$ and normalized noise power is certain in the transmission, when $\lambda_{1}$ is the largest eigenvalue (namely $\boldsymbol{v}_{1}=\boldsymbol{v}_{1 \max }$ is the maximum eigenvector of $\boldsymbol{K}_{t 1}{ }^{\mathrm{H}} \boldsymbol{K}_{t 1}$ ). The signal to noise ratio of combined signal $\boldsymbol{R}_{1}$ is maximized. And from (12), $\boldsymbol{w}_{1}$ is

$$
\boldsymbol{w}_{1}=\boldsymbol{w}_{t 1} T^{-1}=\left(\boldsymbol{T}^{-1} \boldsymbol{K}_{1} \boldsymbol{v}_{1 \max }\right)^{\mathrm{H}} \boldsymbol{T}^{-1} .
$$

So the combined signal and equivalent channel response matrix according to antenna port 1 can be express in (20).

$$
R_{1}=w_{1} Y_{1}, Q_{1}=w_{1} K_{1} .
$$

\section{(3) SSD processing}

Utilizing the antenna based combining method for antenna port $i(i=2,3 \cdots R)$, and regard the output ( equivalent signal and equivalent channel response ) as input of SSD in 3GPP LTE protocol. The output of the SSD is the receiving result, and the receiving of Fig.1 is accomplished.

Summary of section 2 : The combining receiving procedure is (1) constructing antenna port based signal model; (2) Noise power transform; (3) MRC combination; (4) MIMO SSD demodulation.

\section{BER Simulation Result}

By using Matlab2012a, we add relay node and different reception methods in the LTE transmission simulation system of [14]. The simulation system is configured as below:

(1)The transmitter : $1 / 3$ Turbo code, $1.4 \mathrm{M}$ transmitting band, 2 antenna transmit diversity MIMO scheme, transmit power normalized to 1 , and eNB and relay take have the same transmission scheme;(2)The receiver: 2 receiving antenna, perfect channel estimation, SSD demodulation, HARQ scheme;(3)Channel using PedA1simulation model.

We simulated our receiving combination method, selective combination method and equal gain combination method in three situations and got the result curves.

A. Access link channel is better than direct link channel

We simulated our receiving combination method, selective combination method and equal gain combination method in

By setting the noise power in access link and direct link, the access link channel is better than direct link, and the signal received from access link before processing satisfies SNR:-5 $\mathrm{dB}$ to $10 \mathrm{~dB}$, the signal received from direct link before processing satisfies SNR: worse than access link by $5 \mathrm{~dB}$.

Finally the BER simulation of different receiving method is Fig.3. From Fig.3, when these 3 methods obtained the same BER of $10^{-3}$, selective combination reception has $5 \mathrm{~dB}$ gain than equal gain combination reception, and our combination receiving method has $2 \mathrm{~dB}$ gain improvement than selective combination reception.

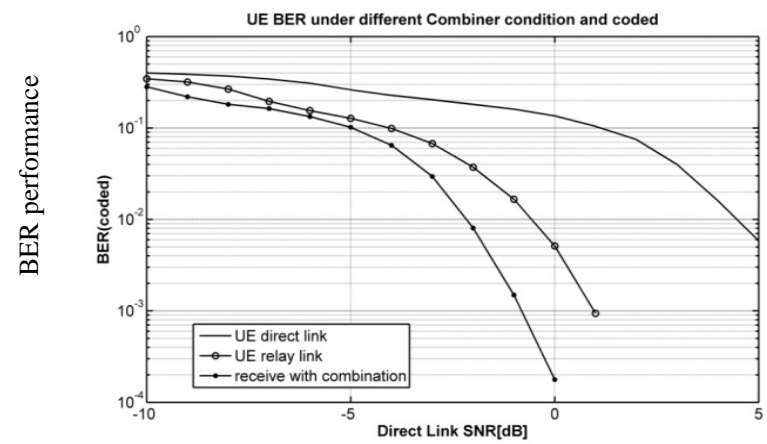

SNR of Direct Link signal before processing (dB)

Fig. 3 BER result when Access link is better than direct link channel

B. Access link channel isn't better than direct link channel

By setting the noise power, the access link channel is not better than direct link, and the signal received from access link before processing satisfies SNR:- $5 \mathrm{~dB}$ to $10 \mathrm{~dB}$, the signal received from direct link before processing are same .

Finally the BER simulation of different receiving method is Fig.4. From Fig.4, when these 3 methods obtained the same BER of $10^{-2}$, our combination receiving method has about 3 $\mathrm{dB}$ gain improvement than other 2 methods.

C. One of access link channel and direct link channel is extremely bad 
Fig.5 is the simulation result when direct link channel is very bad (signal SNR-15dB), SNR of access link varies from 10 to $5 \mathrm{~dB}$. Fig.6 is the simulation result when access link channel is extremely bad $(-15 \mathrm{~dB})$, direct link signal SNR varies from -10 to $5 \mathrm{~dB}$. From the result, it shows that our receiving method is applicable to bad channels situations.

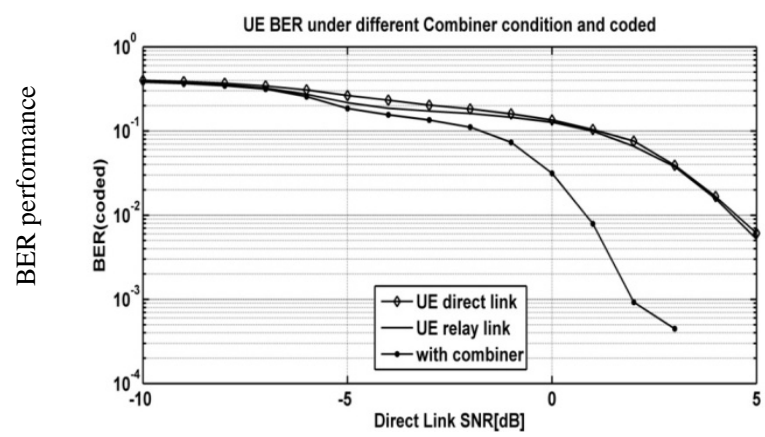

Signal SNR of direct link(dB)

Fig. 4 BER when access link is not better than direct link channel

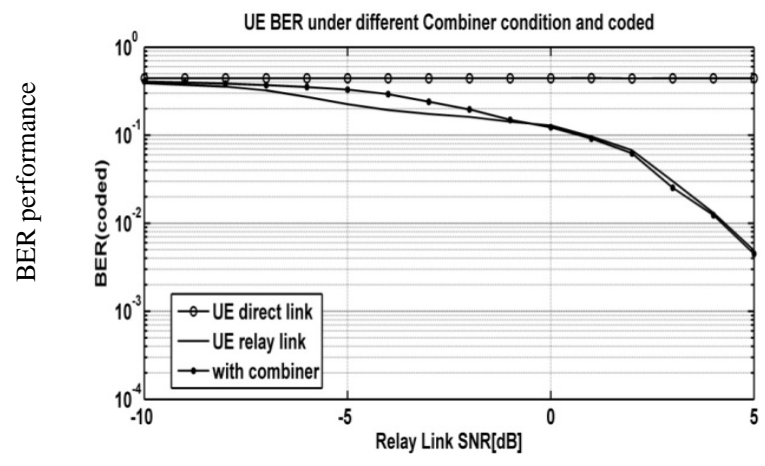

Signal SNR of access link (or relay link) (dB)

Fig. 5 BER result when direct channel is very bad

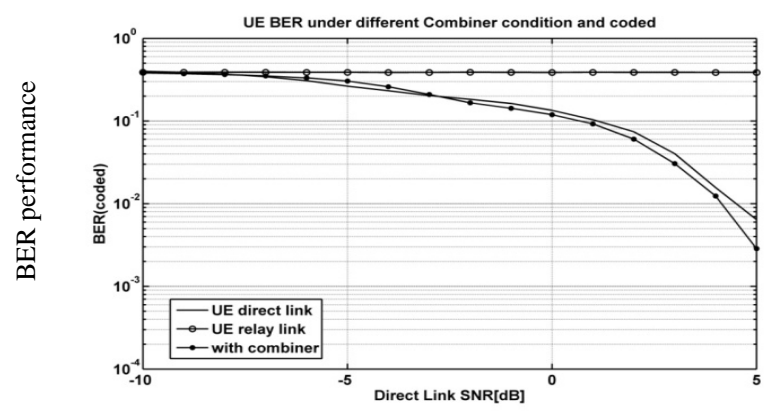

Signal SNR of direct link (dB)

Fig. 6 BER result when access link(or relay link) channel is very bad

\section{Summary}

A receiving model and a receiving method for two hop LTE relay MIMO transmission are proposed in this paper. According to the simulation result, our receiving method has better BER performance than selective and equal gain combining reception method, also it is applicable for extreme channel situation to help relay user improving communication.

\section{Acknowledgment}

Thanks for the support from Beijing science committee project (Z111100066511007) and Beijing Intelligent Safety Production Monitoring Key Laboratory.

\section{References}

[1] Zhang Yong-hong "Selection Maximal Ratio Combining Receiver in VBLAST Systems,” Electronic Science and Technology, 2011, 24 (4): 1-4.

[2] Zhang Sheng-bo, Xia Xiang-gen, Wang Jiang-zhou. "Cooperative Performance and Diversity Gain of Wireless Relay Networks”, Selected Areas in Communications, IEEE Journal, 2012 ,30 (9): 1623-1632.

[3] Liu Yun, Liu Chuan-ju, Zhang Min. "Study on Diversity Gain of Linear Block Code Under Rayleigh Channel,” Computing Engineering, 2012, 38 (7): 90-92.

[4] T K Y Lo. "Maximum ratio transmission," IEEE Trans Commun, Oct, 1999, 47 (2): 1458-1461.

[5] Liu Ling-jia, Chen Rui-hui, S Geirhofer, Shi Zhi-hua, Zhou Yong-xin . "Downlink MIMO in LTE-advanced: SU-MIMO vs. MU-MIMO," Communications Magazine, IEEE, 2012, 50 (2): 140-147.

[6] Dongwoon Bai, Cheolhee Park, Jungwon Lee, etc. "LTE-advanced modem design: challenges and perspectives," IEEE Communications Magazine, 2012, 50 (2): 178-186.

[7] Ketonen J, Juntti M, Cavallaro J R. "Cooperative Communications and Networking,” NewYork, Cambridge, 2008: 178-210.

[8] R H Y Louie, Li Yong-hui, H A Suraweera, B vucetic. "Performance analysis of beamforming in two hop amplify and forward relay networks with antenna correlation," Wireless Communications, IEEE Transactions, 2009,8 (6): 3132-3141.

[9] Liang Yang, Alouini M S. "On the BER and capacity analysis of MIMO MRC systems with channel estimation error," Wireless and Mobile Computing, Networking and Communications (WiMob) ,2011 IEEE 7th International Conference, Stockholm, 2011, 193-198.

[10] Y Chen, C Tellambura. "Performance analysis of maximum ratio transmission with imperfect channel estimatiom," Communications Letters, IEEE, 2005,9 (4): 322-324.

[11] A Maaref, S Aïssa. "Closed-form expressions for the outage and ergodic Shannon capacity of MIMO MRC systems," IEEE Trans Commun, 2005 ,5 (37): 1092-1095.

[12] P A Dighe, R K Mallik, S Jamuar. "Analysis of transmit-receive diversity in Rayleigh fading,” IEEE Trans Commun, 2003,51 (4): 694703.

[13] Shi Hai-dong, Tian Zeng-bin, Wang Xiao-dong. "Performance analysis of distributed MIMO MRC system in composite fading channels," International Conference on Systems and Informatics, Yantai, 2012, 1441-1444.

[14] Meidlinger M, Qi Wang. "Performance evaluation of LTE advanced downlink channel estimators," International Conference on Systems Signals and Image Processing, Vienna, 2012, 252-255.

[15] Li Guang-qiu, Ge Jian-run, Meng Xiang-nan. "Error rate performance analysis of cooperative communication systems with MIMO MRC and imperfect CSI,” Journal of Circuits and Systems, 2011 ,16 (4): 80-85. 